\title{
Health-related quality of life after laparoscopically assisted vaginal hysterectomy: Is uterine weight a major factor?
}

\author{
Pao-Ling Torng ${ }^{1}$, Wen-Chun Chang ${ }^{1}$, Jing-Shiang Hwang ${ }^{2}$, Wen-Chiung Hsu ${ }^{1}$, Jung-Der Wang ${ }^{3,4, *}$, \\ Su-Cheng Huang ${ }^{1}$, Chen-Fang $\mathrm{Chen}^{3}$ \& Ta-Chen $\mathrm{Su}^{4}$ ** \\ ${ }^{1}$ Department of Obstetrics and Gynecology, National Taiwan University Hospital, National Taiwan \\ University College of Medicine, Taipei Taiwan; ${ }^{2}$ Institute of Statistical Science, Academia Sinica, Taipei \\ Taiwan; ${ }^{3}$ Institute of Occupation Medicine and Industrial Hygiene, College of Public Health, National \\ Taiwan University, Rm. 719, No. 17, Xu-Zhou Road, Taipei 10020, Taiwan; ${ }^{4}$ Department of Internal \\ Medicine, National Taiwan University Hospital, National Taiwan University College of Medicine, No. 7 , \\ Chung-Shan South Road, Taipei 10020, Taiwan(E-mail: tachensu@ha.mc.ntu.edu.tw; jdwang@ha.mc.ntu. \\ edu.tu)
}

Accepted in revised form 31 August 2006

\begin{abstract}
Objective: To assess uterine size, symptom severity, and hemoglobin level as determinants of health-related quality of life (HRQOL) in women subsequently undergoing laparoscopically assisted vaginal hysterectomy (LAVH). Methods: Sixty-one consecutive women with uterine leiomyoma or adenomyosis undergoing LAVH were studied using a prospective cohort design. The Chinese version of the Uterine Fibroid Symptom and Quality of Life (UFS-QOL) questionnaire was assessed preoperatively. The Taiwan brief version of the World Health Organization Quality of Life (WHOQOL-BREF) questionnaire and a selfassessment of the perceived health status were assessed preoperatively and 1 day, 1 week, 12 weeks, and 18 months postoperatively. Results: Women with a greater uterine weight did not report a greater severity of symptoms than those with lower uterine weight. Women with more severe symptoms had lower preoperative hemoglobin levels and were usually younger. Their perceived health status and their scores in physical domain of WHOQOL-BREF were also significantly lower, indicating a poorer HRQOL. The mixed-effects model found that a normal (higher) baseline hemoglobin level and a greater symptom severity were associated with a significant improvement of HRQOL in the physical domain of WHOQOL-BREF following LAVH. Conclusions: Preoperative symptom severity, but not uterine weight, was associated with long-term improvement in HRQOL after LAVH. Women with severe symptoms could be considered for LAVH before development of anemia.
\end{abstract}

Key words: LAVH, UFS-QOL, Uterine adenomyosis, Uterine leiomyoma, WHOQOL-BREF

\section{Introduction}

Hysterectomy is the most common non-pregnancy-related surgical procedure performed on women. During the last 20 years, laparoscopy has become an important surgical tool in the evaluation and treatment of gynecologic disease. Laparoscopically assisted vaginal hysterectomy
(LAVH) is currently accepted as a safe and efficient way to manage benign uterine disease with a lower risk of trauma and morbidity [1-5]. More and more women in Taiwan currently choose LAVH instead of the traditional abdominal hysterectomy $(\mathrm{AH})$ to treat their disease. $\mathrm{LAVH}$ is preferable to $\mathrm{AH}$ due to the additional advantages of LAVH over AH, such as the superior cosmetic 
effect, reduced postoperative pain and discomfort, less requirement for analgesia, shorter hospital stay, and more rapid recovery $[1-3,5]$.

Common indications for hysterectomy are prolonged or heavy menstrual bleeding resulting in anemia, symptoms due to the effect of enlarged uterine mass such as pain and urinary symptoms, and/or reproductive dysfunction [6]. These indications are related clinically to benign gynecologic diseases such as leiomyoma and adenomyosis [7]. Decision-making regarding surgery is mainly based on these subjective symptoms, which are usually alleviated thereafter. However, these subjective symptoms cannot be easily or objectively measured, which highlights the need to apply and validate the assessments of health-related quality of life (HRQOL). Studies have shown that symptoms such as bleeding, pelvic pain, dyspareunia, and fatigue are abolished or significantly reduced after surgery [8-10]. Sexual function, psychiatric symptoms and HRQOL are also substantially improved in patients undergoing hysterectomy [11-14]. Ample clinical, evidence-based data have reported that laparoscopic surgery (i.e., a closed procedure) results in greater improvement in HRQOL than an open procedure $[15,16]$. The main purpose of a hysterectomy is to improve the patient's health status. Therefore, the usual decision for surgery is based on an improvement in symptoms (including anemia), and in turn, health status or HRQOL. However, the association of these symptoms with measurable clinical parameters before surgery has not been fully elucidated.

Uterine size, one of the main clinical parameters, has been widely debated as a criterion for removal of the uterus. Before the era of laparoscopic surgery, the American College of Obstetricians and Gynecologists (ACOG) advocated hysterectomy for women with fibroids equal to or exceeding that the comparable size of a 12-week gestation. However, this guideline was not universally supported and hysterectomy for uterine leiomyoma was strongly suggested only in symptomatic patients who did not desire future fertility [17]. After the advent of laparoscopic surgery, ACOG suggested that LAVH could be considered as the treatment of choice instead of $\mathrm{AH}$ for patients with a large uterine size [18]. With the benefits of laparoscopic surgery and the increased amount of clinical data available, it is worthwhile to revisit the debate that uterine size should be used as an indication for surgical intervention. However, performing LAVH with an enlarged uterus is always a clinical challenge. Recently, we reported a novel surgical technique of uterine artery identification and ligation through retrograde tracking of the umbilical ligament (RUL) during the LAVH procedure [19]. Using this technique, we are able to perform LAVH in enlarged uteri with fewer complications, including less blood loss and shorter operative time [19]. With fewer complications, these patients may benefit more with an immediate improvement in health status postoperatively. Furthermore, RUL has allowed us to include cases of enlarged uterine size for the study of empirical improvements in HRQOL after LAVH. Herein, in addition to uterine size, we measured other preoperative clinical parameters, such as symptom severity and preoperative hemoglobin level, which may serve as potential predictors for improvement in HRQOL postoperatively. Patients were assessed preoperatively and four times during 18 months of follow up care.

\section{Methods}

\section{Participants and data collection}

Patients treated with LAVH were evaluated prospectively at the National Taiwan University Hospital, Taipei. From March through December 2003, 61 consecutive patients suffering from nonmalignant uterine conditions, such as uterine leiomyoma or adenomyosis, and undergoing treatment with LAVH using the standard procedure of uterine artery ligation [19] were recruited for the study. Three women with extremely enlarged uteri were assigned for abdominal hysterectomy before surgery, and thus were excluded from our study. None of the patients included during this study were diagnosed with major depressive disorder, according to the Diagnostic and Statistical Manual of Mental Disorders, 4th edition (DSM-IV) diagnostic criteria. Pertinent information, including age, marital status, gravidity, parity, menopausal status, presenting diagnosis, and preoperative hemoglobin level were recorded. After surgery, each patient was provided 
routine postoperative care. The pathologic diagnosis, uterine weight, and in-hospital adverse events were recorded. This study was approved by the Institutional Review Board of the National Taiwan University Hospital. Informed consent was obtained from each participant at the time of enrollment.

Prior to surgery, the patient's symptom severity and the impact of symptoms on HRQOL were assessed with the Chinese version of the Uterine Fibroid Symptom and Quality of Life Questionnaire (UFS-QOL) from the Cardiovascular and Interventional Radiology Research and Education Foundation [20]. The UFS-QOL is a conditionspecific questionnaire, and was used to evaluate a patient's condition preoperatively in this study. Because menstruation-related symptoms usually did not exist after removal of the uterus, we did not measure the UFS-QOL after operation. The Taiwan brief version of the World Health Organization Quality of Life (WHOQOL-BREF; [21]) was completed preoperatively and again postoperatively at 1 day, 1 week, 12 weeks, and 18 months. A self-assessment of perceived health status was performed using a $100 \mathrm{~mm}$ scale measurement. It was measured preoperatively and at the four aforementioned time periods postoperatively. Questionnaires before surgery and 1 day after surgery were administered during hospitalization. The 1 week and 12 weeks postoperative questionnaires were completed during outpatient clinic follow-ups. Patient's status at 18 months was determined by mailed questionnaires, with telephone follow-up for those patients who did not respond in a timely fashion.

During administration of the questionnaire, a recall period of 3 months was recommended for the UFS-QOL because of the variability in the severity and duration of menses in women with uterine leiomyoma and adenomyosis. The recall period was 2 weeks for the WHOQOL-BREF questionnaire.

\section{Questionnaires}

The UFS-QOL has been reported to have high reliability, internal consistency, and good intraclass correlation coefficients [20], and was used successfully in several different hospitals for patients with myoma $[22,23]$. It consists of 37 items with a five-level Likert scale [20]. It was designed with two major scales: (1) a symptom severity scale (UFS8) (8 items) and (2) a HRQOL scale (HRQL total) (29 items) with 6 subscales: concern, activities, energy/mood, control, selfconsciousness, and sexual function. The Chinese version of the UFS-QOL was developed by independent forward and backward translations and was approved by the original developers.

The Taiwan brief version of the WHOQOLBREF was developed from the short form of the WHOQOL with an addition of two national items [21]; it includes four domains (viz., physical, psychological, social, and environmental) of HRQOL and has been demonstrated to have good reliability and validity in Taiwan [21].

\section{Statistical analysis}

The raw scores of symptom severity (UFS8), each subscale and HRQL total were transformed into a 0-100 scale following the scoring manual of UFSQOL [20]. For UFS8, lower scores indicated less severe symptoms. But for UFS-QOL, HRQL total and WHOQOL-BREF, lower scores indicated a greater symptom impact on HRQOL and therefore a poorer HRQOL. For perceived health status, lower scores indicated a worse self-perception of heath status.

Patient characteristics were assessed in subgroups of patients based on USF8, hemoglobin level and uterine mass. Based on clinical significance, UFS8 was divided at a score of 40 , hemoglobin levels at $12 \mathrm{~g} / \mathrm{dL}$ and uterine weight at 300 g. Descriptive analyses of the demographic, clinical variables, and different domains of HRQOL were performed. The correlation between clinical variables such as preoperative uterine weight, symptom severity, hemoglobin level, and age were evaluated by linear regression analysis. The progressive changes in perceived health status and physical domain of the WHOQOL-BREF by different groups of patients were calculated. The statistical analyses for postoperative assessments vs. baseline in each group of patients were performed by using paired $t$-tests.

The internal consistency reliability in the Chinese version of the UFS-QOL questionnaire was assessed by Cronbach's $\alpha$. Concurrent validity between each subscale on the UFS-QOL and each 
domain on the WHOQOL-BREF questionnaires were examined by Pearson's correlation coefficients.

The measured scores in the HRQOL domains at all time intervals were fitted with a linear mixedeffects model with covariates, including uterine weight, UFS8 index, preoperative hemoglobin level, age, marital status, parity, and education. The rational use of a mixed model assumes repeated measurements to have a random effect, which corresponds more so to the repeated measurements of the WHOQOL-BREF for every participant under fixed effects of potential confounders, including age, uterine weight, UFS8, etc., as shown in Table 3. Statistically, it was generally more efficient than simply taking the mean and can possibly improve the power of detection with limited study participants. Specifically, $Y_{j t}$ is the measured score of every domain in the WHOQOL-BREF for the $j$ th participant $(j=1,2, \ldots, n)$ at the $t$ th time $(t=0,2 / 7,1,12$, and 78 weeks $)$, and therefore represent the improvement of WHOQOL-BREF score after LAVH. Assuming that the overall trend is at most quadratic over the 78 weeks, the mixedeffects model is given as follows:

$$
\begin{aligned}
& Y_{j t}=\left(\alpha_{0}+a_{j 0}\right)+\left(\alpha_{1}+a_{j 1}\right) t+\left(\alpha_{2}+a_{j 2}\right) t^{2} \\
& +\beta_{1} \text { Uterine weight }_{j}
\end{aligned}
$$

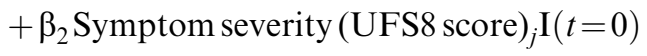

$$
\begin{aligned}
& +\beta_{3} \mathrm{Hb}_{j} \mathrm{I}(t=0) \\
& +\beta_{4} \mathrm{Age}_{j}+\beta_{5} \text { Marriage }_{j} \text { (married, others) } \\
& +\beta_{6} \operatorname{Parity}_{j}(0,1-6) \\
& +\beta_{7} \text { Education }_{j}(\text { groups } 1,2 \text {, and } 3)+\varepsilon_{j t}
\end{aligned}
$$

The $\beta$ 's are fixed regression coefficients associated with the covariates. The trend is determined by three fixed coefficients $\left(\alpha_{k}\right)$ and random coefficients $\left(a_{j k}\right)$, which are assumed to be normally distributed and lack correlation, with a mean of zero and constant variances $\left(\sigma_{k}^{2}\right)$. The error term, $\varepsilon_{j t}$, is also normal with a mean of zero and a constant variance. The intraclass correlation, given by $\rho=\left(\varepsilon_{j \mathrm{t}}, \varepsilon_{j s}\right)$, is added for the repeated measurements.

$p$-Values less than 0.05 were considered statistically significant, except with respect to multiple comparisons, in which statistical significance was considered at $p$-values less than 0.01 . The statistical analysis was carried out using the Statistical Analysis System (SAS), version 8.0 (SAS Institute, Cary, NC).

\section{Results}

Participants were largely married women who had at least completed a middle school education. Most of them had at least one child and were premenopausal at the time of surgery (Table 1). The average age of women upon entry into the study was 46.4 years (range: $36-70$ years). The average uterine weight was $354.9 \mathrm{~g}$ (range: $95-890 \mathrm{~g}$ ), with $50.8 \%$ greater than $300 \mathrm{~g}$ and $18 \%$ greater than $500 \mathrm{~g}$. The average UFS 8 was 42.9 (range: 9.4-100), and the average preoperative hemoglobin level was $11.4 \mathrm{~g} / \mathrm{dL}$ (range: $4.5-14.3 \mathrm{~g} / \mathrm{dL}$ ). The operative complications were minor. Specifically, small bladder lacerations occurred in two patients and were repaired laparoscopically. Both patients were nulliparous and the extirpated uteri weighed 190 and $390 \mathrm{~g}$, respectively. The patient with the lesser uterine weight underwent myomectomy twice before LAVH was performed. Of the 61 patients undergoing $\mathrm{LAVH}$, the percentage of questionnaire response rates on the 2 nd day, 1st week, 12th weeks, and 18th month follow-up visits were $91.8 \%, 82 \%, 59 \%$, and $57.4 \%$, respectively. The percentage response rate in at least one of the last two follow-ups, either the 12 weeks or 18 th months, was $83.6 \%$. Patients not eligible or unwilling to participate in one of the last two follow-up questionnaires were similar in their baseline characteristics as compared to those who completed the follow-up studies.

By fitting linear regression models separately for the variables of interest, we found that symptom severity correlated significantly and negatively with lower uterine weight (Figure 1a), lower baseline hemoglobin levels (Figure 1c), and younger age (Figure 1e), indicating that patients with a smaller uterine weight, younger age, or more anemic usually suffered from more severe symptoms. There was no significant correlation between the baseline hemoglobin level and the uterine weight (Figure 1b).

Scores in each subscale or domain were stratified according to uterine weight ( $>300 \mathrm{~g}$ vs. $\leq 300 \mathrm{~g}$ ), 


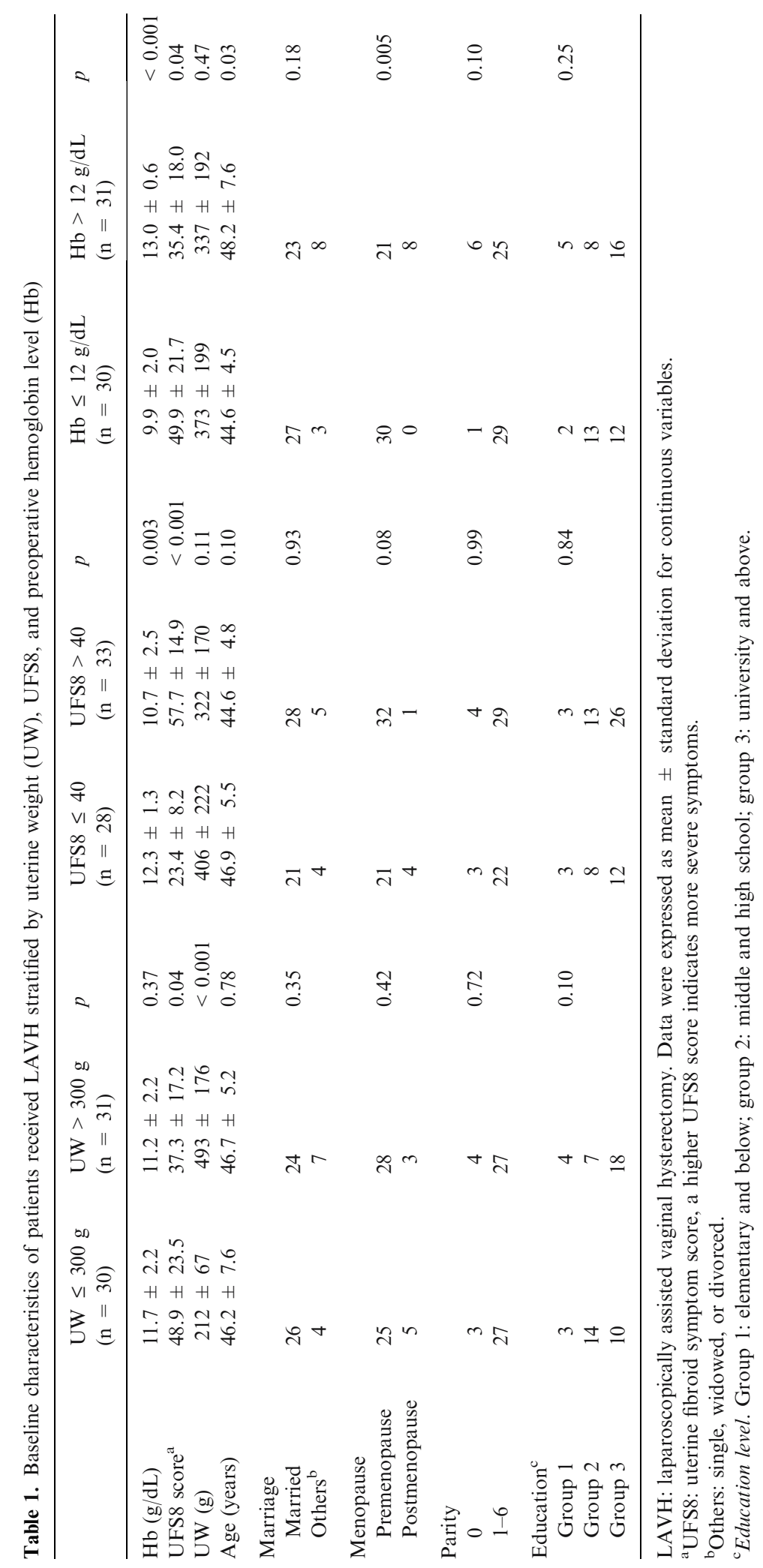



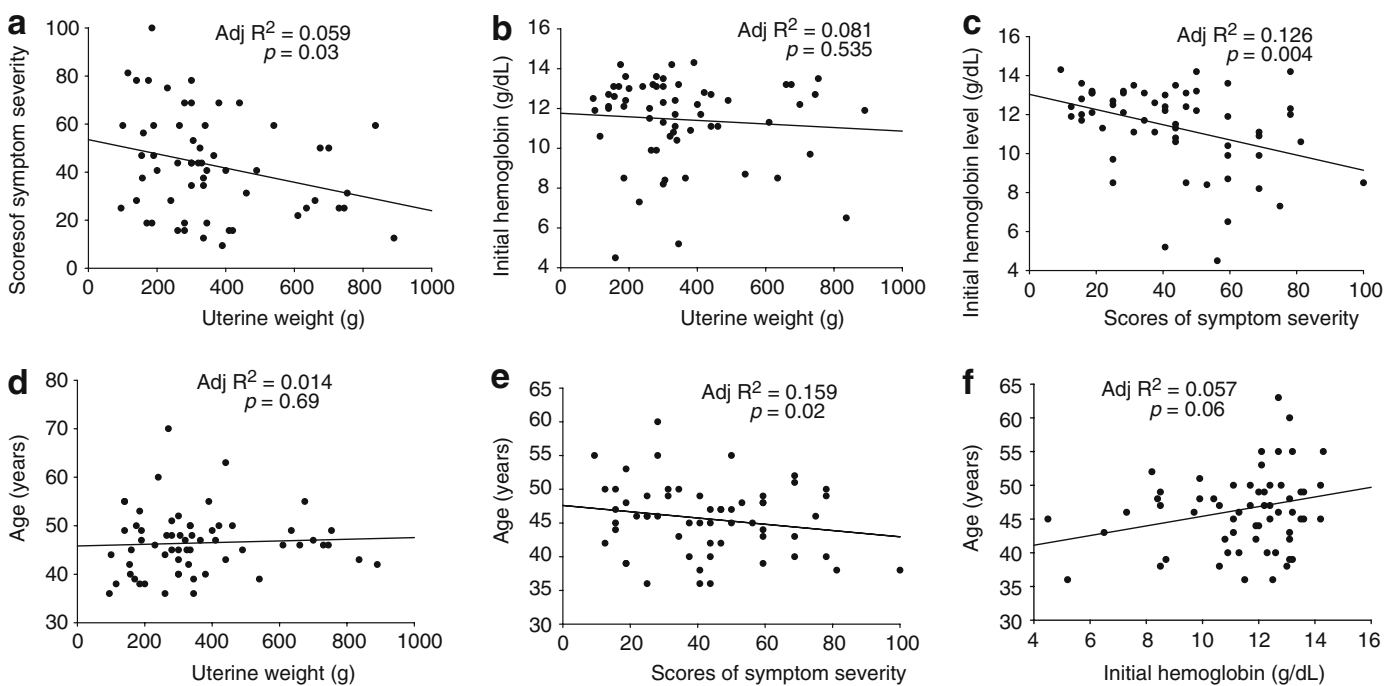

Figure 1. Scatter plots and fitted lines from simple linear regression analysis between different clinical and demographic variables, including uterine weight, score of symptom severity, preoperative hemoglobin level, and age.

symptom severity (UFS $>40$ vs. $\leq 40$ ), or baseline hemoglobin level $(\leq 12 \mathrm{~g} / \mathrm{dL}$ vs. $>12 \mathrm{~g} / \mathrm{dL})$. Preoperatively, patients with lower uterine weight had significantly lower scores (i.e., poor HRQOL) in the physical and environmental domains of the WHOQOL-BREF than patients with a greater uterine weight. Patients with more severe symptoms (UFS8 > 40) had significantly lower scores (i.e., poor HRQOL) on all of the UFS-QOL subscales, except for self-consciousness. Patients with more severe symptoms had significantly worse perceived health status and poorer HRQOL in the physical domain of WHOQOLBREF. Patients with a lower baseline hemoglobin level had significantly poorer HRQOL on all subscales of the UFS-QOL, except for the control and sexual function, than those with a higher baseline hemoglobin level. There was no difference in perceived health status in subgroups based on uterine weight and hemoglobin level.

Using paired comparisons by each postoperative assessment vs. baseline, the perceived health status and the physical domain of WHOQOL-BREF in patients with a lower uterine weight and more severe symptoms (UFS8 > 40) showed significant improvements in the follow-up assessments (Figure 2a, b, d, and e). In women with higher uterine weights, perceived health status improved at the follow-up assessments at 12 and 78 weeks (Figure 2a). Perceived health status improved in all patients regardless of hemoglobin levels after discharge (Figure 2c). However, significant improvement on HRQOL in the physical domain of WHOQOL-BREF was found only in women with normal hemoglobin level (Figure 2f).

The Chinese version of the UFS-QOL used herein was first used in Asia, and we tested its internal consistency reliability. Though our sample size was inadequate, the result seemed satisfactory. The Cronbach's $\alpha$ for subscales of self-consciousness and control were 0.77 and 0.84 , respectively, while those of all the other four subscales and the HRQL total were above 0.92 . This supported the feasible application of UFS-QOL in ethnic Chinese. Since the UFS-QOL was no longer used postoperatively, we studied the correlation coefficients between the domains of the WHOQOL-BREF and the subscales of the UFS-QOL. We found high correlations of several of UFS-QoL subscales with the physical domain of the WHOQOL-BREF that lend support for concurrent validity between these two questionnaires (Table 2).

The results of the mixed-effects model are summarized in Table 3, which showed no significant effect of different uterine weights on all of the domains of the WHOQOL-BREF. Rather, patients with more severe symptoms or a normal (higher) hemoglobin level were more likely to report a significant improvement in the scores of physical domain of the WHOQOL-BREF. 

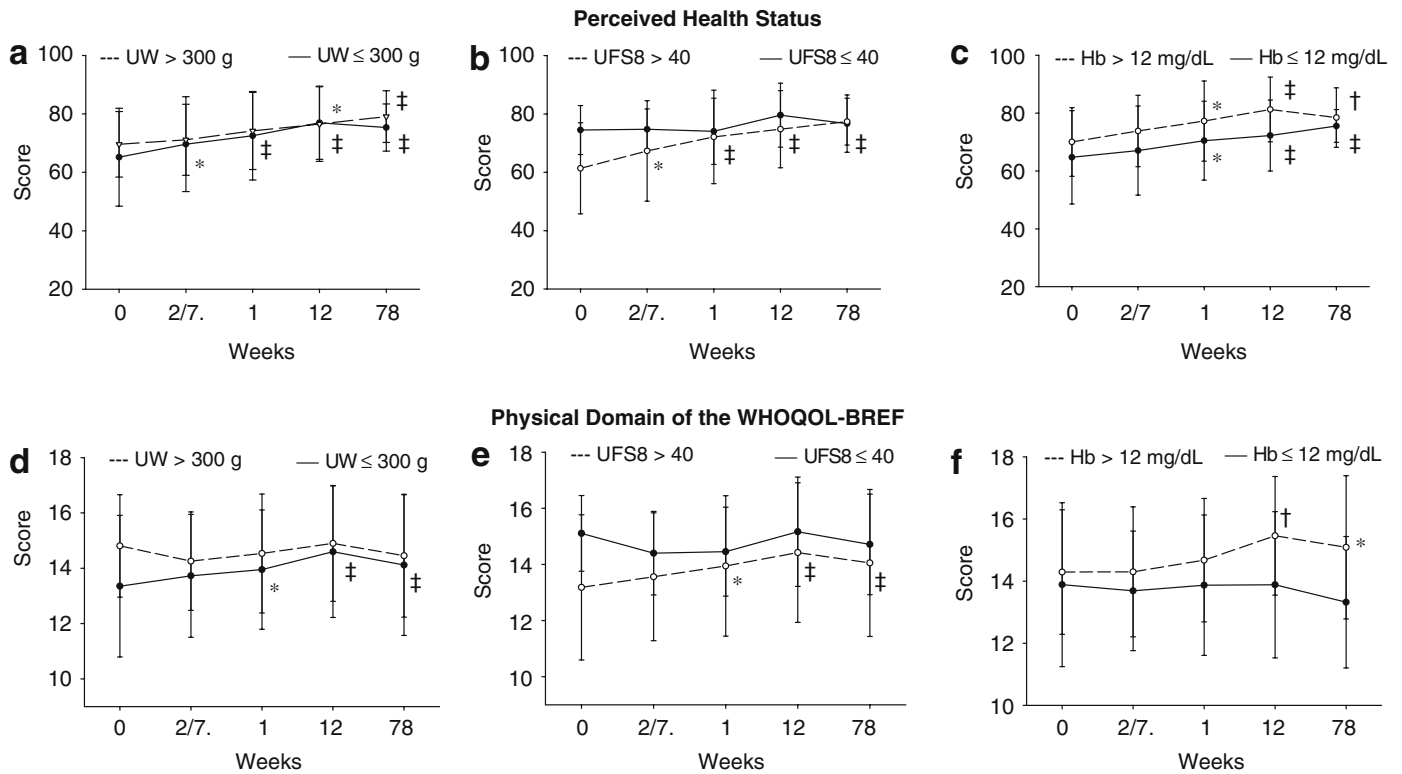

Figure 2. The serial follow-up assessments of perceived health status (a)-(c) and physical domain of the WHOQOL-BREF (d)-(f) by different groups of patients: either by uterine weight, symptom severity, or preoperative hemoglobin level. The bar represents the range of the mean \pm 1 standard deviation; (a and d) groups according to uterine weights (UW), ( - ) $\leq 300 \mathrm{~g}$, and (- - ) $>300 \mathrm{~g}$; (b and e) groups according to scores of symptom severity (UFS8), (-) UFS8 $\leq 40$, and (- - -) UFS8 > 40; (c and f) groups according to preoperative hemoglobin level $(\mathrm{Hb}),(-) \leq 12 \mathrm{~g} / \mathrm{dL}$, and $(--)>12 \mathrm{~g} / \mathrm{dL}$; week 0 represents assessment before surgery, weeks $2 / 7,1,12$, and 78 represent surveys at four different follow-up time points postoperatively. ${ }^{*} p<0.05$, $\dagger p<0.01, \ddagger p<0.001$ indicate the significance levels of paired comparison between scores at follow-up time point and baseline assessments.

\section{Discussion}

The enormous number of hysterectomies performed around the world has resulted in a corresponding increase in health care expenditures, which causes concern in countries such as Taiwan with national health insurance programs and global budget systems [25]. Thus, a set of reasonable criteria are necessary to allow for an unambiguous decision-making process with regards to having such a common surgical procedure. Currently, the criteria used for hysterectomies are rather subjective. Variables based on clinical parameters are warranted for objective decision-making prior to proceeding with hysterectomy. We studied the baseline characteristics of patients undergoing LAVH and found that uterine weight, per se, was not directly correlated with symptom severity and the improvement of HRQOL postoperatively. However, symptom severity and the baseline hemoglobin level were significantly associated with the improvement of HRQOL in the physical domain of WHOQOL-BREF after LAVH. These findings seem counterintuitive and deserve careful scrutiny before enacting conclusions which may influence public health policy.

To rule out potential biases that might be caused by a cross-sectional survey just before and after surgery, we designed this longitudinal study with 18 months of follow-up to ensure a consistent and long-lasting improvement of HRQOL after LAVH, as shown in Figure 2. The extension of our study to 18 months caused a high attrition rate; however, the number of cases studied was still adequate for a linear mixed-effects model analysis. This analysis was used to control other potential confounders of repeated measurements (Table 3). In addition, nearly one-half of our patients had a uterine weight greater than $300 \mathrm{~g}$, and thus provided us with an excellent opportunity to assess whether uterine weight really matters when making this type of decision. Using linear regression analysis, uterine weight seemed to be negatively associated with symptom severity, as shown in 
Table 2. Correlation coefficients between UFS-QOL subscales and HRQL total, and four domains of Taiwan version of WHOQOL-BREF

\begin{tabular}{llllllll}
\hline & \multicolumn{7}{l}{ UFS-QOL } \\
\cline { 2 - 7 } WHOQOL-BREF & Concern & Activities & Energy/mood & Control & Self-consciousness & Sexual function & HRQL total \\
\hline Physical & $0.559 \dagger$ & $0.595 \dagger$ & $0.649 \dagger$ & $0.619 \dagger$ & $0.412^{*}$ & 0.250 & $0.582 \dagger$ \\
Psychological & $0.406^{*}$ & $0.378^{*}$ & $0.424 \dagger$ & $0.368^{*}$ & 0.321 & 0.1607 & $0.360^{*}$ \\
Social & $0.341^{*}$ & $0.338^{*}$ & $0.478 \dagger$ & $0.418 \dagger$ & 0.250 & 0.094 & 0.321 \\
Environment & 0.323 & 0.264 & $0.436 \dagger$ & 0.310 & $0.348^{*}$ & -0.116 & 0.298 \\
\hline
\end{tabular}

UFS-QOL: uterine fibroid symptom and quality of life; HRQL: health-related quality of life; WHOQOL-BREF: the brief version of the World Health Organization Quality of Life questionnaire.

$*_{p}<0.01$ for significant tests of correlation coefficient.

$\dagger p<0.001$ for significant tests of correlation coefficient.

Table 3. Estimated coefficients \pm standard errors of the linear mixed-effects model for scores in every domain of Taiwan version of the WHOQOL-BREF

\begin{tabular}{|c|c|c|c|c|}
\hline Estimated effects & Physical & Psychological & Social & Environment \\
\hline Intercept & $10.32 \pm 2.48 \dagger$ & $11.94 \pm 2.68 \dagger$ & $10.50 \pm 2.38 \dagger$ & $10.13 \pm 2.39 \dagger$ \\
\hline Time (weeks) & $0.07 \pm 0.03^{*}$ & $-0.00 \pm 0.03$ & $0.05 \pm 0.02$ & $0.00 \pm 0.02$ \\
\hline UW (g) & $0.00 \pm 0.00$ & $0.00 \pm 0.00$ & $0.00 \pm 0.00$ & $0.00 \pm 0.00$ \\
\hline UFS8 (scores) & $-0.02 \pm 0.01 *$ & $-0.01 \pm 0.01$ & $-0.00 \pm 0.01$ & $0.00 \pm 0.01$ \\
\hline $\mathrm{Hb}(\mathrm{g} / \mathrm{dL})$ & $0.09 \pm 0.03^{*}$ & $0.01 \pm 0.03$ & $0.03 \pm 0.03$ & $-0.02 \pm 0.03$ \\
\hline Age (years) & $0.06 \pm 0.05$ & $0.02 \pm 0.05$ & $0.04 \pm 0.05$ & $0.09 \pm 0.05$ \\
\hline Married (Yes vs. no) & $-0.40 \pm 1.03$ & $0.41 \pm 1.11$ & $-0.00 \pm 0.99$ & $0.37 \pm 0.99$ \\
\hline Parity (1-6 vs. 0 ) & $1.21 \pm 1.14$ & $0.62 \pm 1.23$ & $1.83 \pm 1.10$ & $0.42 \pm 1.10$ \\
\hline \multicolumn{5}{|l|}{ Education $^{\mathrm{a}}$} \\
\hline Group 1 vs. 3 & $-0.08 \pm 0.90$ & $0.89 \pm 0.97$ & $1.02 \pm 0.86$ & $0.58 \pm 0.86$ \\
\hline Group 2 vs. 3 & $-0.76 \pm 0.57$ & $-1.06 \pm 0.61$ & $-0.87 \pm 0.55$ & $-0.45 \pm 0.55$ \\
\hline AIC & 843.2 & 878.0 & 816.8 & 786.7 \\
\hline
\end{tabular}

UW: uterine weight; UFS8: symptom severity score; Hb: preoperative hemoglobin level; WHOQOL-BREF: the brief version of the World Health Organization Quality of Life; AIC: Akaike's information criterion [24].

$* p<0.01$.

$\dagger p<0.001$.

${ }^{a}$ Education level. Group 1: elementary and below; group 2: middle and high school; Group 3: university and above.

Figure 1a; but it was not associated with the baseline hemoglobin level, as shown in Figure 1b. And there was no improvement in HRQOL based on different uterine weights according to the linear mixed-effects model. Instead, the baseline hemoglobin level and the severity of symptoms measured by the UFS-QOL were consistently associated with improvement in the physical domain of the WHOQOL-BREF after LAVH. This association persisted after control of potential confounding factors through modeling. Thus, we may tentatively conclude that the baseline hemoglobin level and severity of symptoms are the two most important factors regarding decision-making for LAVH in patients with adenomyosis or uterine myoma.

There are some limitations in the current study. First, the condition-specific UFS-QOL questionnaire was not used postoperatively since menstruation-related symptoms do not occur after hysterectomy. The generic WHOQOL-BREF questionnaire was used during the follow-up study. However, it is generally less sensitive to changes than the disease-specific UFS-QOL questionnaire. For example, participants with a low level of hemoglobin only reported improvement in perceived health status but not in physical domain of the WHOQOL-BREF. The correlation coefficients 
between the UFS-QOL and WHOQOL-BREF questionnaires at the initial visit were almost satisfactory in the physical domain, as shown in Table 2. In addition, the WHOQOL-BREF was found to be feasible in assessing changes in HRQOL assessments during the postoperative follow-up. The last two measurements were performed at 12 weeks and/or 18 months postoperatively to minimize the potential learning effect among patients. Moreover, we used the perceived health status as additional information for preoperative measurement and postoperative follow-up.

The second limitation of this study was that WHOQOL-BREF is a generic questionnaire that contains general HRQOL items instead of disease specific conditions. Thus, impairment of HRQOL resulted from symptoms related to an enlarged uterine mass such as bearing down sensation, urinary frequency or disfigure on appearance might not be detected. However, since these symptoms are usually more tolerable than other symptoms cause by massive, irregular bleeding or severe pain, it is not surprising that a generic questionnaire cannot necessarily reflect such an improvement. As the WHOQOL-BREF was demonstrated to effectively show a significant difference between a normal population and patients with human immunodeficiency virus infection [26], epilepsy [27], end-stage renal disease [28], and low back pain [29] in the Taiwanese, it may also detect a difference between patients with uterine weights greater than $300 \mathrm{~g}$ vs. uterine weights lesser than $300 \mathrm{~g}$, albeit in the opposite direction (Figure 2). The finding could explain why our study participants with a small uterus had more severe symptoms that caused them to seek an operation. Figure 2 also indicated generally no significant differences in perceived health status and scores in the physical domain between patients with uterine weights greater than vs. less than $300 \mathrm{~g}$. Thus, we tend to consider that the effect caused by an enlarged uterine mass might have been less of a concern in our patients. Third, there were only two cases with mild complications. Had the selection of participants included more women with numerous previous abdominal operations and adhesions or an enlarged uterus, the results might not have been as good because of other complications, such as bladder injuries, massive blood loss, or even conversion to laparotomy.
Finally, the preoperative questionnaire was completed in the hospital setting, which might result in a higher level of anxiety, thus interfering with HRQOL measurements. Therefore, our conclusion on the improvement of HRQOL postoperatively must be more conservative.

By the mixed-effects model, uterine weight was not found to be associated with the improvement of the patient's HRQOL postoperatively. To perform LAVH in patients with a large uterine size is technically more difficult and therefore might result in more complications or blood loss. Using the RUL procedure, we were able to perform LAVH with enlarged uteri weighing as much as $890 \mathrm{~g}$ in this study with a lesser amount of blood loss [19]. The uterine weight and the time spent in surgery in our study are comparable to other reports involving LAVH [30-32]. Even so, we would not recommend LAVH for an extremely large uterus. Moreover, the results of our study also suggested that asymptomatic patients with large uteri would not benefit from LAVH.

The WHOQOL-BREF did not demonstrate a significant difference between anemic and nonanemic patients at a hemoglobin level of $12 \mathrm{~g} / \mathrm{dL}$ (Figure 2). This could be due to the limited sample size in our study. However, with the statistical application of the mixed-effects model during a longer period of follow-up, we did see a significant effect of hemoglobin level on improvement of HRQOL after LAVH. Interestingly, we found women with normal initial hemoglobin levels improved significantly in the physical domain of the WHOQOL-BREF at 12 weeks and 18 months postoperatively, while no improvement was observed in women with a lower baseline hemoglobin level (Figure 2f). Because women with lower baseline hemoglobin levels improved similarly as those with normal hemoglobin levels in their perceived health status over the 18 months' follow-up (Figure 2c), we suspected that chronically anemic women might be accustomed to limited living activities, which might result in a lower demand for physical domain of HRQOL, as was also reported by other investigators [33, 34]. Thus, women with severe symptoms seeking for LAVH should not wait until a great drop of hemoglobin level.

Symptom severity was significantly associated with the improvement of HRQOL in physical 
domain after LAVH in our mixed-effects model. In addition, the improvement of perceived health status and HRQOL in the physical domain in patients with more severe symptoms (UFS8 > 40) were consistent over the 18 months' follow-up. This suggested that the decision for LAVH based on the patient's severity of symptoms and baseline hemoglobin level were promising, both immediately and persistently after surgery.

\section{Conclusion}

This study demonstrated that, based on the improvement of HRQOL after LAVH, the decision criteria for LAVH in patients with benign uterine disease, such as uterine leiomyoma or adenomyosis, should rely on severity of symptoms rather than uterine weight. We hope that women with severe symptoms should not be delayed for LAVH until a great drop of hemoglobin levels, because they are expected to have a significant improvement in HRQOL in the physical domain of WHOQOL-BREF after LAVH.

\section{Acknowledgements}

This work was supported by grant NHRI-EX949204PP from the National Health Research Institutes and grant NTUH095-000400 from National Taiwan University Hospital.

\section{References}

1. Nezhat F, Nezhat C, Gordans S, Wilkins F. Laparoscopic versus abdominal hysterectomy. J Report Med 1992; 37 : 247-250.

2. Olsson J, Ellstrom M, Hahlin M. A randomized prospective trial comparing laparoscopic and abdominal hysterectomy. Br J Obstet Gynaecol 1996; 103: 345-350.

3. Raju K, Auld B. Randomized prospective study of laparoscopic vaginal hysterectomy versus abdominal hysterectomy. Br J Obstet Gynaecol 1994; 101: 1068-1071.

4. Marana R, Busacca M, Zupi E, Garcea N, Paparella P, Catalano G. Laparoscopic assisted vaginal hysterectomy versus total abdominal hysterectomy: A prospective, randomized, multicenter study. Am J Obstet Gynecol 1999; 180: $270-275$.

5. Schütz K, Possover M, Merker A, Michels W, Schneider A. Prospective randomized comparison of laparoscopicassisted vaginal hysterectomy (LAVH) with abdominal hysterectomy $(\mathrm{AH})$ for the treatment of the uterus weighing $>200$ g. Surg Endosc 2002; 16: 121-125.

6. Schaffer JI, Word A. Hysterectomy - still a useful operation. N Engl J Med 2002; 347: 1360-1362.

7. Stewart EA. Uterine fibroids. Lancet 2001; 357: 293-298.

8. Carlson KJ, Miller BA, Fowler FJ Jr. The Maine Women's Health Study. I. Outcomes of hysterectomy. Obstet Gynecol 1994; 83: 556-565.

9. Kjerulff KH, Langenberg PW, Rhodes JC, Harvey LA, Guzinski GM, Stolley PD. Effectiveness of hysterectomy. Obstet Gynecol 2000; 95: 319-326.

10. Kjerulff KH, Rhodes JC, Langenberg PW, Harvey LA. Patient satisfaction with results of hysterectomy. Am J Obstet Gynecol 2000; 183: 1440-1447.

11. Hartmann KE, Ma C, Lamvu GM, Langenberg PW, Steege JF, Kjerulff KH. Quality of life and sexual function after hysterectomy in women with preoperative pain and depression. Obstet Gynecol 2004; 104: 701-709.

12. Thakar R, Ayers S, Georgakapolou A, Clarkson P, Stanton S, Manyonda I. Hysterectomy improves quality of life and decreases psychiatric symptoms: A prospective and randomised comparison of total versus subtotal hysterectomy. Br J Obstet Gynaecol 2004; 111: 1115-1120.

13. Kuppermann M, Summitt RL Jr, Varner RE, et al. Sexual functioning after total compared with supracervical hysterectomy: A randomized trial. Obstet Gynecol 2005; 105: 1309-1318.

14. Rannestad T. Hysterectomy: Effects on quality of life and psychological aspects. Best Pract Res Clin Obstet Gynaecol 2005; 19: 419-430.

15. Korolija D, Sauerland S, Wood-Dauphinee S, et al. Evaluation of quality of life after laparoscopic surgery: Evidence-based guidelines of the European Association for Endoscopic Surgery. Surg Endosc 2004; 18: 879-897.

16. Garry R, Fountain J, Brown J, et al. Evaluate hysterectomy trial: A multicentre randomised trial comparing abdominal, vaginal and laparoscopic methods of hysterectomy. Health Technol Assess 2004; 8: 1-154.

17. Friedman AT, Haas ST. Should uterine size be an indication for surgical intervention in women with myomas. Am J Obstet Gynecol 1993; 168: 751-755.

18. American College of Obstetricians and Gynecologists.. Appropriate use of laparoscopically assisted vaginal hysterectomy. Obstet Gynecol 2005; 105: 929-930.

19. Chang WC, Torng PL, Huang SC, et al. Laparoscopically assisted vaginal hysterectomy with uterine artery ligation through retrograde umbilical ligament tracking. J Minim Invasive Gynecol 2005; 12: 336-342.

20. Spies JB, Coyne K, Guaou Guaou N, Boyle D, SkyrnarzMurphy K, Gonzalves SM. The UFS-QOL, a new diseasespecific symptom and health-related quality of life questionnaire for leiomyomata. Obstet Gynecol 2002; 99 : 290-300.

21. Yao G, Chung CW, Yu CF, Wang JD. Development and verification of validity and reliability of the WHOQOLBREF Taiwan version. J Formos Med Assoc 2002; 101: 342-351.

22. Spies JB, Myers ER, Worthington-Kirsch R, Mulgund J, Goodwin S, Mauro M, FIBROID Registry Investigators. 
The FIBROID Registry: Symptom and quality-of-life status 1 year after therapy. Obstet Gynecol 2005; 106: 1309-1318.

23. Stewart EA, Rabinovici J, Tempany CM, et al. Clinical outcomes of focused ultrasound surgery for the treatment of uterine fibroids. Fertil Steril 2006; 85: 22-29.

24. Bozdogan H. Model selection and Akaike's information criterion: The general theory and its analytical extension. Psychometrika 1987; 52: 345-370.

25. Hsueh YS, Lee SY, Huang YT. Effects of global budgeting on the distribution of dentists and use of dental care in Taiwan. Health Serv Res 2004; 39: 2135-2153.

26. Fang CT, Hsiung PC, Yu CF, Chen MY, Wang JD. Validation of the World Health Organization quality of life instrument in patients with HIV infection. Qual Life Res 2002; 11: 753-762.

27. Liou HH, Chen RC, Chen CC, Chiu MJ, Chang YY, Wang JD. Health related quality of life in adult patients with epilepsy compared with a general reference population in Taiwan. Epilepsy Res 2005; 64: 151-159.

28. Shih FJ, Lee PH, Wang JD, et al. Changes in quality of life and working capacity before and after kidney transplantation. Transplant Proc 1999; 31: 1981-1984.

29. Horng YS, Hwang YH, Wu HC, et al. Predicting healthrelated quality of life in patients with low back pain. Spine 2005; 30: 551-555.

30. Milad MP, Morrison K, Sokol A, Miller D, Kirkpatrick L. A comparison of laparoscopic supracervical hysterectomy vs laparoscopically assisted vaginal hysterectomy. Surg Endosc 2001; 15: 286-288.
31. Kohler C, Hasenbein K, Klemm P, Tozzi R, Schneider A. Laparoscopic-assisted vaginal hysterectomy with lateral transsection of the uterine vessels. Surg Endosc 2003; 17: 485-490.

32. Hwang JL, Seow KM, Tsai YL, Huang LW, Hsieh BC, Lee C. Comparative study of vaginal, laparoscopically assisted vaginal and abdominal hysterectomies for uterine myoma larger than $6 \mathrm{~cm}$ in diameter or uterus weighing at least $450 \mathrm{~g}$ : A prospective randomized study. Acta Obstet Gynecol Scand 2002; 81: 1132-1138.

33. Lind M, Vernon C, Cruickshank D, et al. The level of haemoglobin in anaemic cancer patients correlates positively with quality of life. Br J Cancer 2002; 86: 1243-1249.

34. Gerson A, Hwang W, Fiorenza J, et al. Anemia and healthrelated quality of life in adolescents with chronic kidney disease. Am J Kidney Dis 2004; 44: 1017-1023.

Address for correspondence: Dr. Ta-Chen Su, Department of Internal Medicine, National Taiwan University Hospital, National Taiwan University College of Medicine, No. 7, ChungShan South Road, Taipei 10020, Taiwan

Tel.: + 886-2-23123456; Fax: + 886-2-23712361

E-mail: tachensu@ha.mc.ntu.edu.tw

Dr. Jung-Der Wang, Professor of College of Public Health, National Taiwan University, Rm. 719, No. 17, Xu-Zhou Road, Taipei 10020 , Taiwan

Tel: + 886-2-3322-8080; Fax: + 886-2-2322-4660

E-mail: jdang@ha.mc.ntu.edu.tw 